\title{
ACCESSIBILITY AND VISUALLY IMPAIRED USERS
}

\author{
António Ramires Fernandes and Jorge Ribeiro Pereira and José Creissac Campos \\ Departamento de Informática, Universidade do Minho \\ Portugal \\ \{antonio.ramires,jrp,jose.campos\}@di.uminho.pt
}

\begin{abstract}
Keywords: Accessibility, Internet, Visually impaired users, talking browsers
Abstract: Internet accessibility for the visually impaired community is still an open issue. Guidelines have been issued by the $\mathrm{W} 3 \mathrm{C}$ consortium to help web designers to improve web site accessibility. However several studies show that a significant percentage of web page creators are still ignoring the proposed guidelines. Several tools are now available, general purpose, or web specific, to help visually impaired readers. But is reading a web page enough? Regular sighted users are able to scan a web page for a particular piece of information at high speeds. Shouldn't visually impaired readers have the same chance? This paper discusses some features already implemented to improve accessibility and presents a user feedback report regarding the AudioBrowser, a talking browser. Based on the user feedback the paper also suggests some avenues for future work in order to make talking browsers and screen readers compatible.
\end{abstract}

\section{INTRODUCTION}

Internet accessibility is still an issue for people with disabilities, in particular for the visually impaired community. Web pages are designed based on the visual impact on the visitor with little or no concern for visually impaired people. HTML is commonly used as a design language and not as a content language.

Two types of complementary directions have emerged to help the visually impaired community:

- Raising the awareness of developers to accessibility issues and providing appropriate methods for web development and evaluation, see for example (Rowan et al., 2000);

- Developing tools that assist visually impaired users to navigate the web.

Sullivan and Matson (Sullivan and Matson, 2000) state that popular press typically reports that $95 \%$ or more of all Web sites are inaccessible to users with disabilities. The study in (Sullivan and Matson, 2000) shows a not so dark picture by performing a continuous accessibility classification. Nevertheless the paper still concludes that "...many Web designers either remain ignorant of, or fail to take advantage of, these guidelines" (Web Content Accessibility Guidelines by WC3).
Sanborn et. al. (Jackson-Sanborn et al., 2002) performed an evaluation of a large number of sites and concluded that $66.1 \%$ failed accessibility testing.

Developing strategies to make web pages more accessible is a solution proposed by many researchers. For instance Filepp et. al. (Filepp et al., 2002) propose an extension for HTML tables. Adding specific tags to HTML tables would allow web page designers to add contextual information.

However these extensions are still a long way from becoming a standard. Furthermore, even when they do become a standard, there is still the issue of getting web designers to follow them. The W3C Guidelines have been around for quite some time and the results from (Jackson-Sanborn et al., 2002) and (Sullivan and Matson, 2000) show that a large percentage of web designers do not follow the proposed guidelines.

Based on these studies we can conclude that accessibility for the visually impaired community remains an issue. Some solutions have emerged to help this particular community of disabled people providing tools that assist users to navigate the web. These solutions can be categorized regarding their scope:

General Purpose Screen readers

Web Specific Talking Web browsers and Transcoding

Screen readers provide a general purpose solution 
to the accessibility problem for the visually impaired community. Their main advantage is being able to single handedly deal with most software. However this lack of specificity also implies that screen readers are not optimized to deal with any application in particular.

IBM(IBM, 2000) proposes a transcoding system to improve web accessibility which explores similarities between a web page and its neighboring. Common layout structure is then removed, hopefully retaining the important parts of the page.

Talking browsers such as IBM's Homepage Reader ${ }^{1}$, Brookes Talk ${ }^{2}$ (Zajicek et al., 2000) and the AudioBrowser ${ }^{3}$ (Fernandes et al., 2001), on the other hand offer a solution to specific tasks: web browsing and e-mail. Being specific solutions they are unable to deal with other tasks besides web surfing. Nevertheless a specific solution can represent a significant advantage to the user.

Talking browsers and transcoding approaches can take advantage of the underlying structure of the document, and present different views of the same document to the user.

In this paper we shall explore the avenue of solutions based on talking browsers. In section 2 we shall focus mainly on features offered by these applications that improve real accessibility. Section 3 reports on the feedback provided by users of a particular solution, the Audiobrowser. Conclusions and some avenues for future work are proposed in section 4.

\section{TALKING BROWSERS}

In this section some features and issues related to talking browsers web solutions are presented. The focus is mainly on features that provide an added value to the user and not on basic features such as basic navigation.

\subsection{Integration}

An important issue in these solutions is to allow the visually impaired user to share its web experience with regular and near sighted users. All the solutions presented in the first section deal with this issue. The IBM approach is to allow users to resize the window, fonts, and colors. An alternative approach is used both by the Brookes Talk and the AudioBrowser: a distinct area where the text which is being read is displayed in a extra large font. These areas can be resized

\footnotetext{
${ }^{1}$ Available at http://www-3.ibm.com/able/solution_offerings/hpr.html

${ }^{2}$ Available at http://www.brookes.ac.uk/schools/cms/ research/speech/btalk.htm

${ }^{3}$ Available at http://sim.di.uminho.pt/audiobrowser
}

to a very large font making it easier for even seriously near-sighted users to read the text.

All solutions also provide a standard view of the page therefore allowing the visually impaired and near-sighted users to share their experience with regular sighted users. This feature is essential to allow for full inclusion of users with disabilities in the Information Society.

\subsection{Document Views}

The Brookes Talk solution provides an abstract of the web page comprising key sentences. This approach, reduces the amount of text to about $25 \%$ and therefore gives the user an ability to quickly scan the contents of the page.

The AudioBrowser includes a feature that attempts to get the user as quickly as possible to the page's contents. A significant number of web pages has a graphical layout based on two or three columns. The first column usually contains a large set of links, and the main contents is on the second column. Normal reading of one of such pages implies that the user must first hear the set of links before the application reaches the main content itself. In order to get the user as quickly as possible to the main contents of the web page the links are removed. This eliminates most of the content of the first column, therefore the user reaches the main content with just a few key strokes. Figure $1^{4}$ shows the text which will be read using this feature, and figure 2 show the full text of the page as understood by the browser.

It is clear from the figures that using this feature provides a much faster access to the real contents of the web page. In figure 2 the first news item "O presidente do Banco Central..." is not even visible on the window, whereas in figure 1 it appears very near to the top. Another clue is the size of the dragging bar which gives a hint regarding the amount of the text in both views.

Note that table navigation doesn't provide the same functionality since web pages tend to use tables as a design feature and not a content feature. Therefore navigating on a table can be cumbersome.

Based on the same assumption the AudioBrowser allows for the linearization of tables. The reading is currently row based. In this way the artifacts caused by tables tend to be eliminated.

The AudioBrowser also provides a third mode where all the document's hierarchy is presented. In this mode the user is presented with a hierarchical tree where tables, cells and headings are parent nodes of the tree. The text, links and images are the leaf nodes

\footnotetext{
${ }^{4}$ The text in the images is in Portuguese since the $\mathrm{Au}$ dioBrowser is currently being developed for a Portuguese audience only.
} 


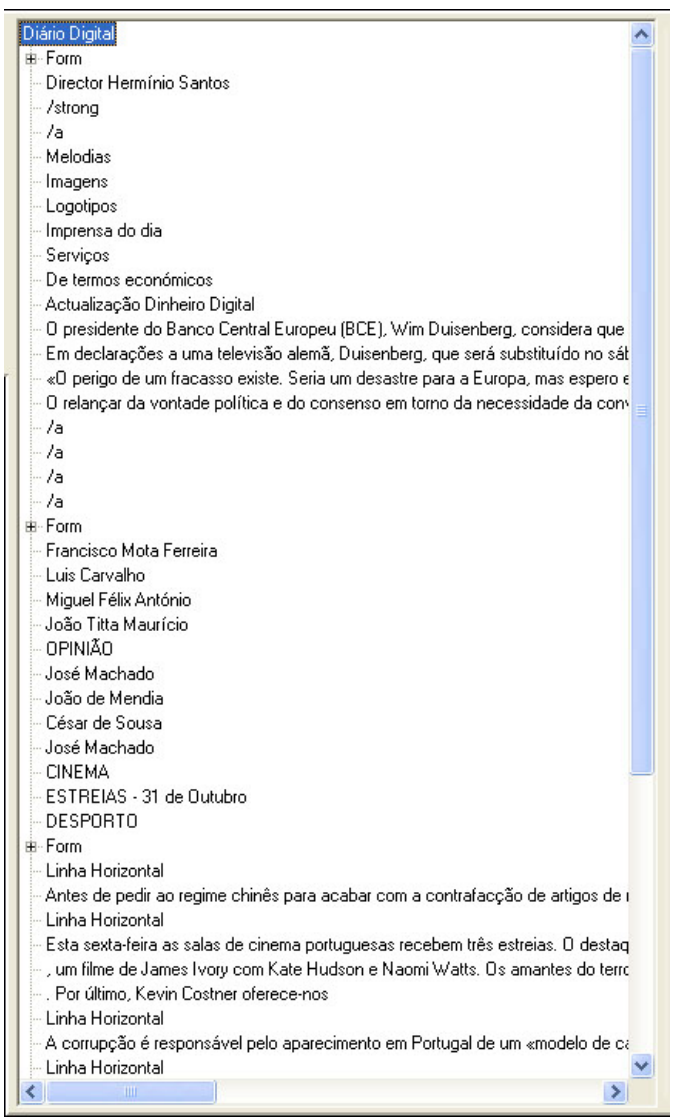

Figure 1: Document View with links removed.

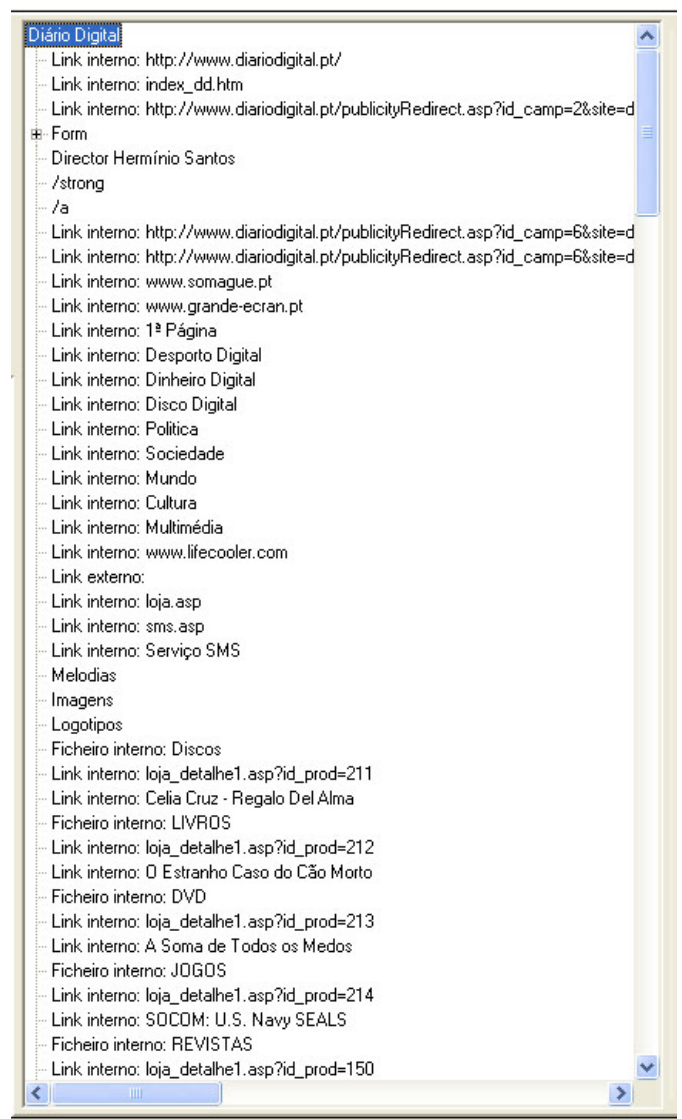

Figure 2: Normal View of the Document.

- internal files;

- external files.

The notion of an external link is not as clear as one might think. The semantics of external imply that the link will lead to a web page outside the group of related pages under the same base address. The base address can be the domain itself, a subdomain, or even a folder.

For instance in geocities, each user is granted an area that has as the base address www.geocities.com/userName. Pages outside this base address, although in the same domain are external to the site from a semantic point of view.

Nevertheless, this classification, even if based only on domains as base addresses, is extremely useful in some circumstances. Consider a web searching engine such as Google. The internal links represent the options of the search engine. Once the search has been performed the external links provide the user with a quick access to the results.

Long documents with anchors are another example. In this case the anchors can provide a sort of table of contents.

\footnotetext{
${ }^{5}$ The images of individuals and commercial brands were deliberately blurred.
} 


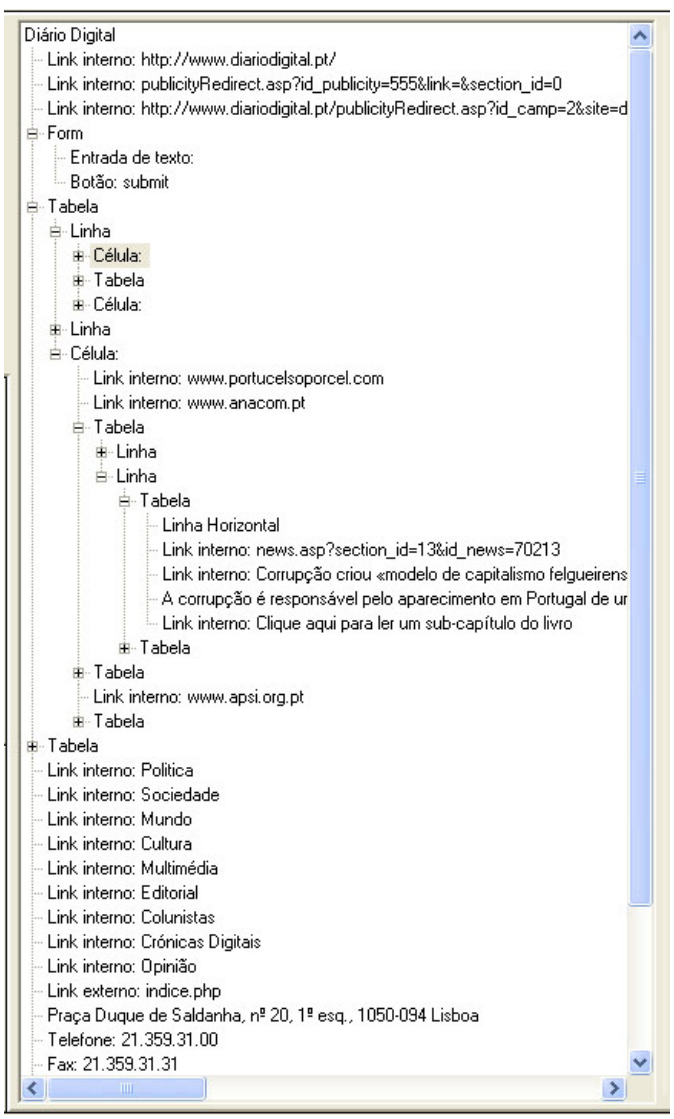

Figure 3: Hierarchical View of the Document

\subsection{Bookmarks}

Bookmarking a web page is a common procedure amongst internet users. However the default bookmarking offered in Internet Explorer is not powerful enough to satisfy fully the visually impaired user, or as a matter of fact for any kind of user.

The idea behind bookmarking is to allow a user to save the address of a web page in order to get back to it latter. This serves a purpose when we consider small web pages, with little volume of text. When we consider large pages, with considerable amounts of text, it may be of use to specify a particular piece of text.

It may be the case that the user wants to bookmark not the page itself, but a specific location in the page, for instance a paragraph. This would allow the user to stop reading a web page at a particular location, and then get back to that location latter without having to start from the beginning of the document again.

Another example where the benefits of bookmarking a specific location of a web page would be of great use is to allow users to skip text in the beginning of the document. A significant number of pages have

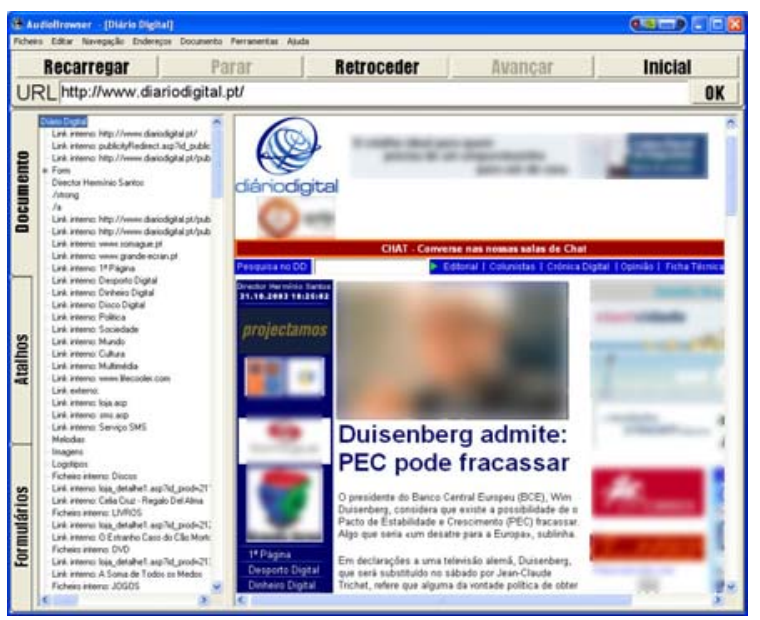

Figure 4: The AudioBrowser

a fixed structure, where only content varies. As mentioned before, it is common to have a left column with links to sections of the site and other sites. Bookmarking the beginning of the main content area, would allow the user to skip the links section when the page is loaded.

Currently the AudioBrowser seems to be the only talking browser that has this feature, including compatibility with Internet Explorer's favorites. Jaws ${ }^{6}$, a popular screen reader, also offers a similar feature called PlaceMarkers.

\subsection{E-mail}

E-mail is no longer restricted to text messages: messages can be composed as a web page. Hence using the same tools as those used for web browsing makes perfect sense. The AudioBrowser is capable of handling e-mail at a basic level, providing the same set of features that are used for web browsing. The AudioBrowser attempts to integrate visually impaired users with regular sighted users, and therefore it uses the same mailbox as Outlook, hence has access to the same received messages. The navigation on the messages is identical to the navigation on a web page, therefore the interface is already familiar to the user.

\section{USER FEEDBACK}

In this section we discuss the feedback we got from users during the project as well as some problems that arose when attempting to perform usability evaluation.

\footnotetext{
${ }^{6}$ Available at http://www.freedomscientific.com/ fs_products/software_jaws.asp
} 
Visually impaired users were involved in the project from its early design stages, and helped in defining the initial requirements for the tool. They were also involved in analyzing early prototypes of AudioBrowser.

To evaluate AudioBrowser's current version we planned a number of usability tests with its users. This process turned out more problematic than what we had initially envisaged. This was partly due to the typical problem of convincing software developers to perform usability evaluation, but mainly due to difficulties in setting up the testing sessions.

Performing usability tests with users is always a difficult and expensive process. We found this even more so when we are considering visually impaired users. As point out in (Stevens and Edwards, 1996), when trying to evaluate assistive technologies difficulties may arise due to a number of factors:

- Variability in the user population - besides the fact that they will all be visually impaired users, there is not much more that can be said to characterize the target audience of the tool (and even that assumption has turned out to be wrong, as it will be discussed later in this section).

One related problem that we faced had to do with the variability of use that different users will have for navigating the web. Due to this, identifying representative tasks for analysis turned out to be a problem.

- Absence of alternatives against which to compare results - in our case this was not a problem since there are other tools that attempt to help users in performing similar tasks.

- Absence of a large enough number of test subjects to attain statistical reliability — this was a major issue in our case. In fact, we had some difficulty in finding users willing to participate in the process. We had access to a reduced number of users and some reluctance was noticed in participating due to (self-perceived) lack of technical skills (a "I don't know much about computers, so I can't possibly help you" kind of attitude). We were, nevertheless, able to carry out a few interviews, and it is fair to state that the users that participated did so enthusiastically.

One further problem that we faced related to identifying exactly what was being analyzed in a given context. The AudioBrowser enables its users to access pages on the web. Suppose that, when visiting some specific page, some usability problem is identified. How should we decided whether the problem relates to the tool or whether it relates to the page that is being accessed? For simple (static) pages this might be easy to decide. For more complex sites, such as web based applications (where navigation in the site becomes an issue), it becomes less clear whether the problem lies with the tool or whether it lies with the site itself.

Due to these issues, we decided to perform informal interviews with the available users, in order to assess their level satisfaction with the tool. More formal usability analysis being left for latter stages.

Even if the reduced number of interviews carried out does not enable us to reach statistically valid conclusions, it enabled us to address some interesting questions regarding the usability of the AudioBrowser tool. The analysis addressed two key issues:

- the core concept of the AudioBrowser - a tool tailored specifically to web page reading;

- the implementation of such concept in the current version of the tool.

Regarding the first issue, the focus on the structure of the text (as represented by the HTML code), instead of focusing on the graphical representation of such text, was clearly validated as an advantage of the AudioBrowser when compared with traditional screen readers. Such focus on the structure of the text enables a reading process more oriented towards the semantic content of the page (instead of its graphical representation), and a better navigation over the information contents of the page.

Regarding the second issue, the tool was found satisfactory in terms of web navigation. However, its implementation as a stand-alone tool (i.e., a tool that is not integrated with the remainder assistive technologies present in the work environment) was found to be an obstacle to its widespread use by the visually impaired community. Aspects such as the use of a separate speech synthesizer from the one used for interaction with the operating systems, create integration problems of the tool regarding the remaining computational environment, and can become difficult barriers for users which are less proficient at the technological level. This is an issue that had not been previously considered, and that clearly deserves further consideration.

Besides the planned usability testing, we have also received reports from regular sighted users who employ the AudioBrowser to check their own web pages regarding structure and accessibility. The hierarchical view of the page provided by the AudioBrowser allows these users to spot potential accessibility and syntax problems that would otherwise be hard to discover. This was a somewhat unexpected application of the tool, and it stresses the difficulties with identifying the end user population of a software artifact. 


\section{CONCLUSIONS AND FUTURE WORK}

On this paper we have presented AudioBrowser, a talking browser that aims at enabling visually impaired users to efficiently navigate the web. AudioBrowser takes advantage of the underlying structure of the document in order to better present its content, and is able to present different views of the same document enabling the user to choose the view that better fits its navigational needs at each moment. This feature is useful not only to visually impaired users, but also for web designers since it allows them to have a broader perspective of the construction of the page. We have received reports of several web designers that are using the AudioBrowser to this effect.

Features and issues related to talking browsers were introduced. In this context some of the main features of the AudioBrowser were described. Focus was on features that provide increased accessibility.

From a usability point of view the main objection, as mentioned previously, is the need to switch back and forth from using the browser to a screen reader, which is still needed for all the other tasks. This is one usability problem that was not foreseen neither by the developers nor the visually impaired people that participated on the AudioBrowser project from the very beginning.

A talking browser and a screen reader are incompatible because they both produce speech, and therefore they can not be working simultaneously.

However talking browsers have several advantages over screen readers and should not be put aside. Talking browsers make it easy to manipulate the web page and present different views, therefore minimizing the scanning time for visually impaired users.

Can talking browsers and screen readers be compatible? A possible solution for this problem could be to have the browser not producing any speech at all. Instead the browser would produce text that would then be read by the screen reader. It may seem absurd to have a talking browser not talking at all, but under this approach the browser is in fact "talking" to the screen reader. In order for this solution to be successful the process would have to be completely transparent to the user. To the user the application would be an enhanced web browser.

A totally different approach would be to have a web service that provides a subset of the functionality of a talking browser solution. This requires a proxy based approach where a web address would be provided. The page would be transcoded in several views in a web server, and all those views would be supplied to a regular browser, for instance using frames. The user would be able to specify which views would be of interest, and each view, plus the original page, would be supplied in a separate frame properly identified.

Although this approach requires extra bandwidth we believe that with the advent of broadband, and considering that the different views have only text, i.e. no images, the overhead would be bearable considering the extra functionality.

Combining a proxy based approach and a talking browser can provide an enhanced web experience, as the transcoded web pages may contain special tags recognized by the talking browser.

\section{ACKNOWLEDGEMENTS}

The research reported in here was supported by SNRIP (The Portuguese National Secretariat of Rehabilitation and Integration for the Disabled) under program CITE 2001, and also FCT (Portuguese Foundation for Science and Technology) and POSI/2001 (Operational Program for the Information Society) with funds partly awarded by FEDER.

\section{REFERENCES}

Fernandes, A. R., Martins, F. M., Paredes, H., and Pereira, J. (2001). A different approach to real web accessibility. In Stephanidis, C., editor, Universal Access in H.C.I., Proceedings of HCI International 2001, volume 3, pages 723-727. Lawrence Erlbaum Associates.

Filepp, R., Challenger, J., and Rosu, D. (2002). Improving the accessibility of aurally rendered html tables. In Proceedings of the fifth international ACM conference on Assistive technologies, pages 9-16. ACM Press.

IBM (2000). Web accessibility transcoding system. http://www.trl.ibm.com/projects/acc_tech/attrans_e.htm.

Jackson-Sanborn, E., Odess-Harnish, K., and Warren, N. (2002). Website accessibility: A study of ada compliance. Technical Report Technical Reports TR-2001-05, University of North CarolinaÜChapel Hill, School of Information and Library Science, http://ils.unc.edu/ils/research/reports/accessibility.pdf.

Rowan, M., Gregor, P., Sloan, D., and Booth, P. (2000). Evaluating web resources for disability access. In Proceedings of ASSETS 2000, pages 13-15. ACM, ACM Press.

Stevens, R. D. and Edwards, A. D. N. (1996). An approach to the evaluation of assistive technology. In Proceedings of ASSETS '96, pages 64-71. ACM, ACM Press.

Sullivan, T. and Matson, R. (2000). Barriers to use: Usability andcontent accessibility on the webŠs most popular sites. In Proceedings on the conference on universal usability, 2000, pages 139-144. ACM Press.

Zajicek, M., Venetsanopoulos, I., and Morrissey, W. (2000). Web access for visually impaired people using active accessibility. In Proc International Ergonomics Association 2000/HFES 2000. 